\title{
Maintenance of Learner's Characteristics by Spreading a Change
}

\author{
Michal Šimún, Anton Andrejko and Mária Bieliková \\ Slovak University of Technology, Faculty of Informatics and Information \\ Technologies, Institute of Informatics and Software Engineering \\ Ilkovičova 3, 84216 Bratislava 4, Slovakia \\ simun.michal@yahoo.com, \{andrejko,bielik\}@fiit.stuba.sk \\ WWW home page: http://www.fiit.stuba.sk
}

\begin{abstract}
Learners have different knowledge, interests and also goals in any educational process. If all the learners are provided the same content the process will probably not meet expected goals. A personalized approach to a learner increases the efficiency of the learning process. It is based on information about the learner that is subject of change. Several adaptive educational systems exist. However, they usually do not consider changes in user behavior and relationships that may exist among contents in order to maintain learner characteristics. In this paper we present a novel method for maintenance of user's characteristics based on spreading activation.
\end{abstract}

\section{Introduction}

Good educational process strongly depends on a combination of the best learning sources aimed at achieving high quality while using the most effective methods of education as possible. Web-based educational applications provide huge amounts of information from various areas that are presented to learners, but usually do not consider the learner's individual knowledge or interest. Such a learner might be provided with content that he/she is not ready to study at that time, is not interested in, or can not find suitable information in large knowledge spaces. Increased efficiency of learning process requires a personalized approach to a learner where characteristics for each learner are modeled in a user oriented model which is used to select the most suitable study material for an individual learner. In this approach the key requirement is to accurately estimate the individual learner's characteristics.

There are several ways to model characteristics in a user model. The most common approaches are stereotype [1] and layered [2,3] user model. In the stereotype approach learners are divided into groups according to the selected difficulty of course. Each learner is assigned to one stereotype only and is provided 
with studying materials assigned to the particular stereotype. The stereotype can be changed after successfully completing the competency for that material (e.g. test, skills demonstration, etc.). In the layered user model the user's characteristics are modeled according to educational documents within a particular course. In this case, evaluation of user characteristics' for a visited document and for related parts of the course (characteristic propagation) is employed. Actualization of a characteristic for the course can be based on defined rules [3] or on analysis of learner's activity [2].

In this paper we focus on modeling user's characteristics while learning. We propose a novel method for maintenance of user's characteristics based on spreading adaptive change in a layered user model.

\section{Adaptive Web-Based Educational System Models}

In the personalization process it is desirable to know the document's attributes (educational content) in an educational course (domain model) and also the user's characteristics (user model). In this paper we provide examples on adaptive learning programming. Individual learner's characteristics are mapped against characteristics of educational materials that express the semantics of educational materials. Therefore, for the domain (and user) model ontology representation [4, 5] is used. Having reusability of the models in mind we divide the domain model into Knowledge item space and Learning objects space as shown in the Fig 1.

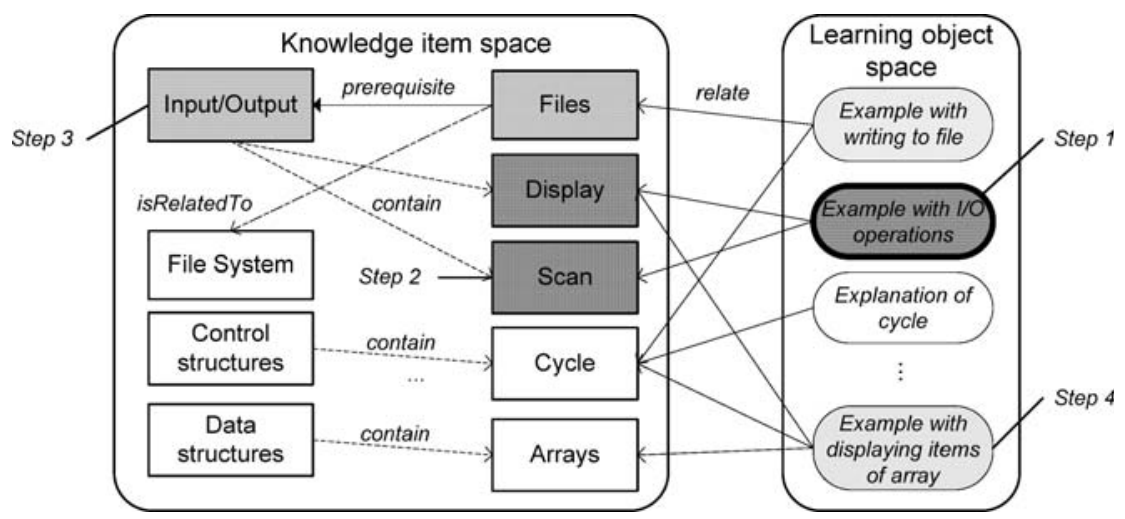

Fig 1. Sequence of steps in process of change of characteristic.

The Learning objects space consists of learning objects and relations between them. This part of the domain model merges with a standard view of the domain model for adaptive web-based systems. A Knowledge Item (KI) represents a topic or a key word that represents key terms of the domain. Its aim is a categorization of available learning objects into knowledge items (using the linkage relate) according to learning goals of particular learning objects. We have added knowledge item level to the domain model to be able to model personalization at the knowledge items level. It allows for a determination of the learner's characteristics for all learning objects related to the knowledge item. Our approach also supports the changing of 
learner's characteristics for the learning objects, even he/she has have not visited them yet. A knowledge item can be connected using relations prerequisite (knowledge items necessary to be understood or known in order to study other subjects), contain (hierarchical content of knowledge item) or isRelatedTo (logically related knowledge items). Personalization in an educational course is based on presentation (recommendation) of appropriate learning objects to an individual learner who is represented in a user model. Furthering the developed user model is influenced by [2]. It consists of records about user's visits, interest and knowledge.

\section{User's Characteristics Maintenance by Spreading a Change}

While a user works with a learning object we obtain his/her characteristics for that learning object (e.g. interest). Since there is a connection between learning objects and other parts of the domain model we incorporate any changes to other related parts of the domain model, even if the user has not worked with these parts yet. The process consists of four steps.

In the first step characteristics for actual learning object (e.g. Example with $\mathrm{I} / \mathrm{O}$ operations in Fig 1) are set. Its characteristics knowledge and interest are changed according to the variety of views and each view defines a strategy for characteristics to be updated. Furthermore, interest and knowledge have assigned characteristic's value (numeric value) and a probability of modeled value (how likely a real value of user's characteristic matches a modeled value of the characteristic). Analyzing learner's activity we directly change following views for actual learning object:

- Feedback: user adjusts interest (knowledge) in a graphical user interface directly;

- Classification: every defined level of the characteristic (e.g. expert) creates a class of classification. According to the selected strategy a model of classification is created that assigns a user into one of the defined classes in regard to user's activity.

In the second step, changes of characteristic's values from actual learning object are applied to knowledge items (knowledge item Display and Scan). For instance, if user studied a learning object focused on cycles it is desirable to increase knowledge for the knowledge item Cycles. Characteristic's value for related KI is computed using all related learning objects that initialized a characteristic with changed view.

Spreading or applying changes of characteristic's values from knowledge items (Scan and Display in our example) to other related parts of knowledge item space (Input/Output and Files) is processed in the third step. A change of a characteristic for knowledge and interest is applied through the modeled relations between knowledge items. Spreading user's characteristic to other knowledge items is based on spreading activation $[7,8]$ where initial energy of a selected node is spread to other nodes, and fades with increasing distance. Energy is a couple (value, probability(value)), where value is a quantified representation of the spread characteristic. Fading is accomplished by sequentially decreased probability of value.

The last step is spreading characteristic's change from the knowledge items to the related learning objects (Example with writing to file). After the change of value and probability of a characteristic for the knowledge item occurs it is necessary to 
process the change of the characteristic for all learning objects. These changes in characteristics occur in the learning objects space that have assigned selected knowledge item. Characteristic's value for related learning object is computed from characteristics of all related knowledge items that have initialized the characteristic.

\section{Conclusion}

A personalized approach to learners provides a way to improve the educational process. We present a method for modeling and maintenance of user's characteristics based on spreading activation. In the first step, characteristics in a source of activation (i.e. learning object a user is working with) are set. Afterwards, spreading is processed to knowledge items related to the selected learning object. Changes in knowledge item characteristic affects also related learning objects.

For evaluation purposes a simple programming course was created. It consists of 16 learning objects and knowledge items are imported from ACM classification. We developed an adaptive web-based educational application that recommends learning objects. It is built on three architectural layers - user, application and data. The data layer contains learning materials and semantics of the course - domain ontology and user's characteristics (in OWL-DL). The application layer is responsible for maintaining of user's characteristics using our method and is implemented in Java SE 6. The user layer is implemented in JRuby and it is responsible for generating views in the educational course and acquiring information about user's activities.

Acknowledgement: This work was supported by the Cultural and Educational Grant Agency KEGA 3/5187/07 and by the Scientific Grant Agency VEGA 1/3102/06.

\section{References}

1. C. Seeberg et al., From User's Needs to Adaptive Documents. In: Proceedings of the Integrated Design \& Process Technology Conference, 2000.

2. E. Schwarz, P. Brusilovsky, J. Eklund., Web-based Education for All: A Tool for Developing Adaptive Courseware. In: Computer Networks and ISDN Systems 30 (1-7), 1998, pp. 291-300.

3. P. De Bra et al., AHA! The Adaptive Hypermedia Architecture. In: ACM Hypertext Conference, Nottingham, UK, 2003, pp. 81-84.

4. P. Brusilovsky, Methods and techniques of adaptive hypermedia. User Modeling and UserAdapted Interaction 6 (2-3), 1996, pp. 87-129.

5. P. Saloun, Z. Velart: Adaptive Hypermedia as a mean for learning programming. In: ICWE, Palo Alto, ACM, 2006, pp. 1-5.

6. A. Andrejko, M. Barla, M. Bieliková. Ontology-based User Modeling for Web-based Information Systems. In G. Knapp et al. (Eds.): Advances in Inf. Sys. Development New Methods and Practice for the Networked Society, Vol. 2, Springer, 2007, pp. 457-468.

7. F. Crestani, P.L. Lee: Searching the Web by Constrained Spreading Activation. In Proc. IEEE Forum on Research and Technology Advances in Digital Library, 1999, pp. 163-170.

8. J. Suchal: Caching Spreading Activation Search. In M. Bieliková (Ed.): IIT.SRC 2007 Student Research Conference 2007. Bratislava, Slovakia, 2007, pp. 151-155. 